\title{
A New Position Location System Using DTV Transmitter Identification Watermark Signals
}

\author{
Xianbin Wang, ${ }^{1}$ Yiyan $\mathrm{Wu},{ }^{1}$ and Jean-Yves Chouinard ${ }^{2}$ \\ ${ }^{1}$ Communications Research Centre Canada, 3701 Carling Avenue, Ottawa, Canada ON K2H 8S2 \\ ${ }^{2}$ Department of Electrical and Computer Engineering, Laval University, Canada QC G1K 7P4
}

Received 30 May 2005; Revised 30 January 2006; Accepted 9 March 2006

\begin{abstract}
A new position location technique using the transmitter identification (TxID) RF watermark in the digital TV (DTV) signals is proposed in this paper. Conventional global positioning system (GPS) usually does not work well inside buildings due to the high frequency and weak field strength of the signal. In contrast to the GPS, the DTV signals are received from transmitters at relatively short distance, while the broadcast transmitters operate at levels up to the megawatts effective radiated power (ERP). Also the RF frequency of the DTV signal is much lower than the GPS, which makes it easier for the signal to penetrate buildings and other objects. The proposed position location system based on DTV TxID signal is presented in this paper. Practical receiver implementation issues including nonideal correlation and synchronization are analyzed and discussed. Performance of the proposed technique is evaluated through Monte Carlo simulations and compared with other existing position location systems. Possible ways to improve the accuracy of the new position location system is discussed.
\end{abstract}

Copyright ( $) 2006$ Xianbin Wang et al. This is an open access article distributed under the Creative Commons Attribution License, which permits unrestricted use, distribution, and reproduction in any medium, provided the original work is properly cited.

\section{INTRODUCTION}

Geographic location information can be retrieved by various infrastructures and technologies. The most popular position location system is the global position system (GPS) based on a constellation of about 24 satellites orbiting the earth at altitudes of approximately 11,000 miles [1]. In Europe, a satellite navigation system named Galileo was deployed by the European Commission and Space Agency based on a 30-satellite constellation, to provide positioning and timing services in 2008 [2]. Uncorrected positions determined from GPS satellite signals produce accuracies in the range of 50 to 100 meters. When using a technique called differential correction, users can get positions accurate to within 5 meters or less. GPS is effective and accurate outdoors, but it works very poorly, if at all, indoors and in urban canyon environments, and a reliable solution is needed to fill these gaps in coverage. Moreover, GPS is vulnerable to jamming and other disruptions from manmade and natural causes. Without a functional backup, widespread disruption of the GPS would be catastrophic for commercial applications, as well as domestic and international security.

New alternative position location systems were recently proposed based on other wireless communication systems, such as cellular networks and wireless LAN. An order issued by the U.S. Federal Communications Commission (FCC) in July 1996 requires that all wireless service providers, including cellular and broadband wireless, provide location information to Emergency 911 (E-911) public safety services [3]. These new FCC E-911 requirements have also boosted research in wireless location techniques. Cellular networks can be used to provide location services, where the mobile stations are located by measuring the signals traveling to and from a set of fixed cellular base stations. However, owing to the low power of each transmitter and narrow bandwidth, position systems based on cellular networks can only achieve very limited accuracy with locationing error often larger than few hundred meters $[4,5]$. With the development of wireless local area networks (LAN), there is an increasing level of interest in developing the technology to geolocate using DSSS/OFDM based wireless LAN systems [6]. Position location system based on wireless LAN is more accurate within the service area of network. However, its application is limited by the network coverage and outdoor locationing information is often unavailable especially for rural areas. Positioning system using television synchronization signals was first proposed in [7]. The major advantage of the television locationing approach is from the low RF frequency, wide band, high transmission power, and broad coverage of DTV transmitters. However, a network of monitor stations has to 
be established to broadcast the timing information for each TV station.

In this paper, a new position location system is proposed based on DTV transmitter identification watermarks. Training sequence in DTV signals might be used for position location and multipath estimation under some circumstances. However, large position location error may be introduced when there is cochannel interference in the DTV signal. In the presence of cochannel interference, multipath estimation is actually the linear combination of the multipath channel responses from all the DTV transmitters on the same channel, since an identical training sequence is used for all cochannel DTV transmitters. In addition, TxID watermark is still needed to identify the transmitter location and propagation time. As a result, the proposed DTV position location system and the subsequent analysis are based on the transmitter identification watermark.

As of May 2005, there are more than 1400 terrestrial digital television (DTV) transmitters in operation in the U.S.A., Canada, and Mexico. The Advanced Television System Committee (ATSC) DTV signals are entirely different from the analog TV signals and have many new capabilities. One interesting new feature of the ATSC signal is that a pseudorandom sequence, used as an RF watermark, can be uniquely assigned to each DTV transmitter for transmitter identification (TxID) purpose [8]. Due to an ever-increasing number of DTV transmitters, the need for transmitter identification is becoming essential since it enables the broadcast authorities and operators to identify the source of in-band interferences. In [8], phase modulation of each TxID sequence can also lead to a robust data transmission approach, which can be used to broadcast the timing and geolocation information for each transmitter. Similar transmitter identification techniques could also be used to DVB-T system in the future. Using relatively simple signal processing, DTV signals from different transmitters can be identified. By varying the phase of the TxID sequence, the timing and location information for each DTV transmitter can also be sent out. Since the locations of the DTV transmitters are known, it is possible to locate the receiver positions when the DTV signals from multiple DTV transmitters can be successfully received and identified.

The proposed position location process using DTV TxID or watermarked signal, can be realized through several steps. (1) Identify the sources for all DTV signals received at one location. This is based on the calculation of cross-correlation between the DTV signals and local TxID sequences. The ATSC field SYNC signal can be used for a quick synchronization of the TxID sequence. (2) Calculate the pseudorange between the receiver and each DTV transmitter. (3) Determine the coordinates of the receiver by solving a nonlinear equation system. When there are more transmitters than needed for location position, optimization techniques can be used to increase the positioning accuracy and reduce the impact of multipath distortion.

The rest of the paper is organized as follows: transmitter identification using RF watermark is elaborated in Section 2. The proposed position location technique using TxID

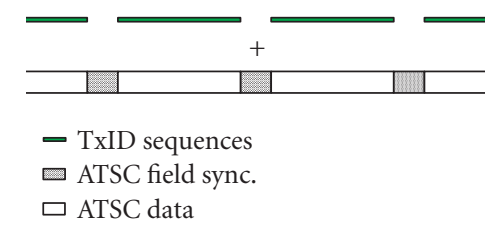

(a)

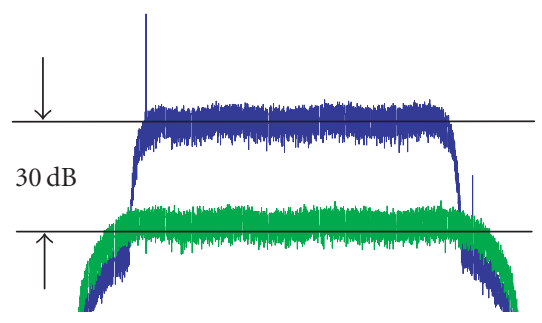

(b)

FIGURE 1: Illustration of the ATSC DTV signal with the embedded spread spectrum sequences. (a) Time domain, (b) frequency domain.

watermark is presented in Section 3. Practical implementation issues including the nonideal cross-correlation function and synchronization for the position location receiver is analyzed and discussed in Sections 4 and 5, respectively. $\mathrm{Nu}-$ merical results for the proposed position technique were presented in Section 6. Example of position location using a nonlinear equation system was also given in this section. The paper is finally summarized in Section 7.

\section{TRANSMITTER IDENTIFICATION FOR DTV}

The proposed position location is achieved based on multiple distance measurements between known reference points, that is, signals from different DTV transmitters have to be identified for the determination of the geographic coordinates. In [9], we proposed a transmitter identification system using embedded pseudorandom sequences. A unique PN sequence is assigned to each individual transmitter in our proposal and different transmitters are identified based on the orthogonality between different sequences. The magnitude of the pseudorandom sequence is carefully selected such that the impact on the DTV reception is negligible. This proposal has been adopted in the ATSC synchronization standard for distributed transmissions [8], where a Kasami sequence with a period of $2^{16}-1$ is used for DTV transmitter identification. The autocorrelation function of this sequence provides $42 \mathrm{~dB}$ dynamic range for transmitter identification $[10,11]$. The principle of the transmitter identification is illustrated in Figure 1 both in frequency and time domain. A similar TxID technique can also be applied to DVB-T systems. Denote the DTV signals for the $i$ th transmitter before and after the injection of the pseudorandom sequence $x_{i}(n)$ as $d_{i}(n)$ and $d_{i}{ }^{\prime}(n)$, respectively. The injected process is

$$
d_{i}^{\prime}(n)=d_{i}(n)+\rho x_{i}(n),
$$


where $\rho$ is a gain coefficient to control the injection level of the identification sequence, which can be different from transmitter to transmitter. However, it will be convenient for the identification process if the gain is the same for all the transmitters. After passing through the channel $h_{i}$, the received signal from the $i$ th transmitter, $r_{i}$, can be formulated as

$$
r_{i}(n)=d_{i}^{\prime}(n) \otimes h_{i}+w(n),
$$

where $w(n)$ is the additive white Gaussian noise (AWGN) of the receiver. To identify the existence of the $i$ th transmitter, the cross-correlation between $r_{i}(n)$ and the locally generated $x_{i}(n)$ has to be calculated:

$$
\begin{aligned}
R_{r x_{i}}(m)= & \sum_{n=0}^{N-1} r(n) x_{i}(n-m) \\
= & \sum_{n=0}^{N-1}\left\{\left[d_{i}(n)+\rho x_{i}(n)\right] \otimes h_{i}+w_{i}(n)\right\} \cdot x_{i}(n-m) \\
= & \rho R_{x_{i} x_{i}} \otimes h_{i}+\left\{\sum_{n=0}^{N-1} d_{i}(n) x_{i}(n-m)\right\} \otimes h_{i} \\
& +\sum_{n=0}^{N-1} w_{i}(n) x_{i}(n-m),
\end{aligned}
$$

where $N$ is the length of the transmitter identification watermark $x_{i}(n)$. The first term on the last line of (3), that is, the autocorrelation function $R_{x_{i} x_{i}}$, exists only when watermark signal $\rho x_{i}(n)$ is found in the received signal. The existence of the $i$ th transmitter can then be determined by the correlation peak in (3) since the watermark signal $\rho x_{i}(n)$ is uniquely associated with the $i$ th transmitter. Equation (3) also indicates that the correlation peak in the first term on the last line undergoes the same attenuation and channel distortion as the DTV signal described by the second term. To evaluate the robustness of transmitter identification process, a simplified AWGN channel model is applied to (3):

$$
\begin{aligned}
R_{r x_{i}}(m)= & A \rho R_{x_{i} x_{i}}+A \sum_{n=0}^{N-1} d_{i}(n) x_{i}(n-m) \\
& +\sum_{n=0}^{N-1} w_{i}(n) x_{i}(n-m),
\end{aligned}
$$

where $A$ is a constant associated with the path loss. Due to the large $N$ for transmitter identification sequence, central limit theorem can be applied to the second and third items in (4), whose variances can be determined as $N A^{2} \sigma_{d}^{2}$ and $N \sigma_{w}^{2}$, where $\sigma_{d}^{2}$ and $\sigma_{w}^{2}$ are the variances of the DTV signal and AWGN noise. The signal-to-interference-and-noise ratio (SINR) of the autocorrelation peak for transmitter iden- tification in (4) can be determined as

$$
\begin{aligned}
\text { SINR } & =10 \log _{10}\left\{\frac{A^{2} \rho^{2} N^{2}}{N A^{2} \sigma_{d}^{2}+n \sigma_{w}^{2}}\right\} \\
& =10 \log _{10} N-10 \log _{10}\left(\frac{A^{2} \sigma_{d}^{2}+\sigma_{w}^{2}}{A^{2} \rho^{2}}\right) \\
& =10 \log _{10} N-10 \log _{10}\left\{\frac{A^{2} \sigma_{d}^{2}\left(1+\sigma_{w}^{2} / A^{2} \sigma_{d}^{2}\right)}{\left.A^{2} \rho^{2}\right\} .}\right.
\end{aligned}
$$

Equation (5) can be further arranged as

$$
\begin{aligned}
\text { SINR }= & 10 \log _{10} N-10 \log _{10}\left(\frac{\sigma_{d}^{2}}{\rho^{2}}\right) \\
& -10 \log _{10}\left(1+\frac{\sigma_{w}^{2}}{A^{2} \sigma_{d}^{2}}\right) .
\end{aligned}
$$

Note that the second term in (6) is the injection ratio of the transmitter identification watermark and $\sigma_{w}^{2} / A^{2} \sigma_{d}^{2}$ is the inverse of the signal-to-noise ratio (SNR) of the received signal, which makes the third item in (6) negligible for any reasonable SNR, that is, $\sigma_{w}^{2} / A^{2} \sigma_{d}^{2} \ll 1$. Because the TxID watermark is inserted at a certain power level proportional to DTV signal, the fixed relationship is maintained after both signals pass through the same multipath channel. Additive Gaussian noise from the receiver has virtually no impact on the TxID process, unless the received signal is significantly weaker than the noise introduced by the receiver, that is, the DTV signal is under the receiver's noise floor. Due to the extremely high transmission power of DTV stations and the short distance between the receiver and transmitter, (6) holds even for the reception sites inside buildings since the excess path losses due to the building penetration is usually around $10 \sim 20 \mathrm{~dB}$ $[12,13]$. As a result, the robustness of the transmitter identification process is dominated by the first two items in (6). For the TxID system in [8], SINR in (6) is $18 \mathrm{~dB}$ when one Kasami sequence is used, or $24 \mathrm{~dB}$ when four Kasami sequences in one field are combined for transmitter identification. Considering the high transmission power of the DTV stations, the coverage limitation for the transmitter identification and the proposed position location is the shape of the earth, rather than the signal strength of the DTV signal.

As we will explain in the next section, four DTV stations are needed for position location purpose. These stations can be on different channels. The position location receiver will scan different TV channels for the DTV stations used for position location. In this situation, the analysis in (1)-(6) can be directly applied to each station. The impact of the cochannel interference from the DTV stations on the same channel with different programs is limited since the coverage of these DTV stations are well separated through the DTV stations planning process. The other scenario for cochannel interference is from DTV stations broadcasting the same program on the same channel due to the deployment of the single frequency network (SFN), in which same content is broadcasted from different stations on the same frequency to save spectrum [14]. Cochannel DTV stations in SFN could be used as 
position location references, since different transmitter identification numbers [8] are assigned to different SFN stations. However, the strength of the TV signals at one given location from different SFN stations can vary significantly due to the different distances from the receiver as well as the different propagation environment. It is therefore very important to analyze the robustness of the transmitter identification under this circumstance since the combined DTV signals from different SFN transmitters will interfere with the transmitter identification process. The overall received signal $r(n)$ can be reformulated as

$$
r(n)=\sum_{i=1}^{M}\left[d_{i}^{\prime}(n) \otimes h_{i}+w(n)\right],
$$

where $M$ is the total number of TV signals from the SFN. The existence of the $j$ th transmitter is unknown without any further identification process. Details of the existence and strength of each specific transmitter at the reception site can be achieved by calculating a correlation function. For instance, cross-correlation between $r(n)$ and $x_{j}(n)$ can indicate the existence and provide strength information about the $j$ th transmitter:

$$
\begin{aligned}
R_{r x_{j}}(m)= & \rho R_{x_{j} x_{j}} \otimes h_{j}+\sum_{i=1, i \neq j}^{M} \rho R_{x_{i} x_{j}} \otimes h_{i} \\
& +\sum_{n=0}^{N-1} \sum_{i=1}^{M}\left[d_{i}(n) \otimes h_{i}\right] x_{j}(n-m) \\
& +\sum_{n=0}^{N-1} w(n) x_{j}(n-m) .
\end{aligned}
$$

With the orthogonal property of the selected pseudorandom sequence, $R_{x_{j} x_{j}}$ can be approximated as a delta Kronecker function. The second term can be neglected since different transmitter identification sequences are orthogonal. The third item in (8) is the combined interference from the SFN DTV signals of the $j$ th transmitter and the other transmitters. Therefore, the received channel response $h_{j}$ from the $j$ th transmitter can be approximated by $R_{r x_{j}}$. An interference analysis for (8) with AWGN channel model lead to

$$
\begin{aligned}
\text { SINR }= & 10 \log _{10} N-10 \log _{10}\left[\frac{\sigma_{d}^{2}}{\rho^{2}}\left(1+\sum_{i=1, i \neq j}^{M} \frac{A_{i}^{2}}{A_{j}^{2}}\right)\right] \\
& -10 \log _{10}\left(1+\frac{\sigma_{w}^{2}}{\sum_{i=1}^{M} A_{i}^{2} \sigma_{d}^{2}}\right) .
\end{aligned}
$$

Comparing (6) and (9), the impact of the cochannel stations in SFN environment can be evaluated by the second term in (9). When the cochannel DTV signal is stronger than the signal from the particular station under identification process, the robustness of transmitter identification is reduced. However, around $10 \mathrm{~dB}$ stronger cochannel DTV signals can be tolerated due to the large margin in the transmitter identification system $[8,9]$. Simple averaging of the transmitter identification results in that the time domain would reduce the impact of the DTV interference by $10 \log _{10} P$, where $P$ is the number of averaging. The complexity associated with averaging is minimal since different DTV signal segments for TxID can be averaged first before the cross-correlation. Further performance improvement can be achieved by the DTV signal cancellation approach. However, the complexity of position location receiver will be increased since the DTV signal has to be reconstructed based on the demodulation result.

\section{TIME-BASED POSITION LOCATION USING TXID SIGNAL}

There are several different approaches to determine the location of receiving devices in a wireless network, ranging from direction-of-arrival detection to calculation of signal strength loss. The technique considered in this paper is based on triangulation. This method derives its name from trigonometric calculations and can be done via lateration, which uses multiple distance measurements between known points, or via angulation which measures an angle or bearing relative to points with known separation. These two techniques are also referred to as direction-based and distancebased techniques. Direction-based techniques measure the angle of arrival (AOA) using antenna array. Because this AOA triangulation technique requires the use of special antennas, it would not be suitable for position location applications. Distance-based techniques involve the measurement and calculation of the distance between a receiver and one or more transmitters whose locations are known. The distancebased technique uses one, or more, of the following signal attributes: signal arrival time, signal strength, and signal phase. If one measures the precise time a signal leaves a transmitter and the precise time the signal arrives at a receiver, he can determine the time of arrival (TOA); the time it takes for the signal to reach the receiver.

Consider four transmitters and the positioning receiver shown in Figure 2. The coordinates of the four transmitters are $\left(x_{1}, y_{1}, z_{1}\right),\left(x_{2}, y_{2}, z_{2}\right),\left(x_{3}, y_{3}, z_{3}\right)$, and $\left(x_{4}, y_{4}, z_{4}\right)$, respectively. For existing DTV transmitters, these coordinates are known to the positioning receivers. With the help of the embedded watermarks and the DTV field sync shown in Figure 3, the propagation time for the DTV signal from each DTV station can be easily determined. Denoting the propagation time from the $i$ th transmitter to the positioning reception point as $t_{i}$, the simplified positioning algorithms without errors can be formulated as

$$
\begin{aligned}
& t_{1} c=\sqrt{\left(x-x_{1}\right)^{2}+\left(y-y_{1}\right)^{2}+\left(z-z_{1}\right)^{2}}, \\
& t_{2} c=\sqrt{\left(x-x_{2}\right)^{2}+\left(y-y_{2}\right)^{2}+\left(z-z_{2}\right)^{2}}, \\
& t_{3} c=\sqrt{\left(x-x_{3}\right)^{2}+\left(y-y_{3}\right)^{2}+\left(z-z_{3}\right)^{2}}, \\
& t_{4} c=\sqrt{\left(x-x_{4}\right)^{2}+\left(y-y_{4}\right)^{2}+\left(z-z_{4}\right)^{2}},
\end{aligned}
$$

where $c$ is the constant for light propagation velocity. Four 


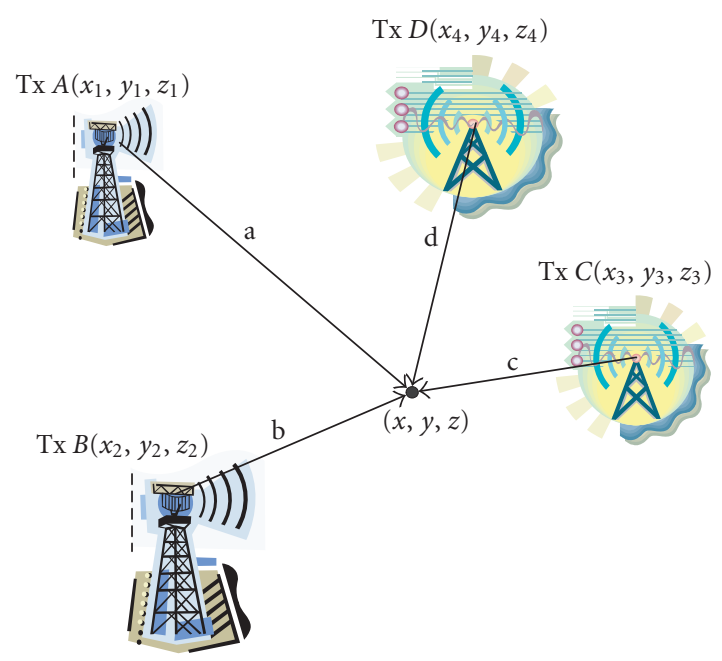

FIgUre 2: Position location system using DTV transmitters.

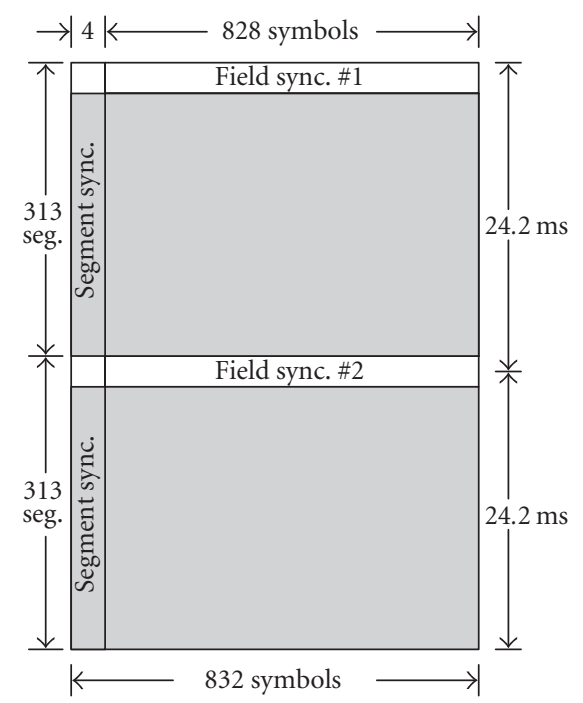

FIGURE 3: One frame of ATSC signal with embedded TxID sequence (shaded region).

transmitters are needed to find the coordinates of the positioning receiver when the absolute propagation time for each transmitter is not available. In this case, what is known from the received signal of the synchronous transmitter network is the relative propagation time, with a common reference timing related to the transmission network. Under this circumstance, (10) can be rewritten as

$$
\begin{aligned}
& t_{1}{ }^{\prime} c=\sqrt{\left(x-x_{1}\right)^{2}+\left(y-y_{1}\right)^{2}+\left(z-z_{1}\right)^{2}}, \\
& t_{2}{ }^{\prime} c=\sqrt{\left(x-x_{2}\right)^{2}+\left(y-y_{2}\right)^{2}+\left(z-z_{2}\right)^{2}}, \\
& t_{3}{ }^{\prime} c=\sqrt{\left(x-x_{3}\right)^{2}+\left(y-y_{3}\right)^{2}+\left(z-z_{3}\right)^{2}}, \\
& t_{4}{ }^{\prime} c=\sqrt{\left(x-x_{4}\right)^{2}+\left(y-y_{4}\right)^{2}+\left(z-z_{4}\right)^{2}},
\end{aligned}
$$

where $t_{i}{ }^{\prime}=t_{i}-\Delta t$ is the absolute transmission time for the $i$ th transmitter with $\Delta t$ being the timing difference between the receiver reference time and the absolute time. The value of $\Delta t$ is unknown but identical for all transmitters since they are all synchronized within the distributed transmitter network. The pseudorange equation in (11) can be solved by the technique in [15] without errors or by linearizing techniques in [16] in the presence of errors.

As indicated in (11), the relative propagation time from each transmitter to the positioning receiver has to be determined. The existence and the strength of each specific transmitted signal $r_{j}$ from the $j$ th transmitter at a given reception site can be achieved by calculating correlation functions. For example, the correlation between $r(n)$ and a locally generated identification signal $x_{j}(n)$ can provide the existence and strength of the $j$ th transmitter using (8). Due to the orthogonal property of the selected sequence, $R_{x_{j} x_{j}}$ can be approximated as a delta function. The second and third terms in (8) are only noise-like sequences from the in-band DTV signals of the same transmitter and other transmitters. Therefore, the channel response $h_{j}$ from the $j$ th transmitter can be approximated by $R_{r x_{j}}$, that is,

$$
R_{r x_{j}}(m)=A h_{j}+\text { noise, }
$$

where $A$ is a constant determined by $R_{x_{j} x_{j}}$ and the gain coefficient $\rho$. The channel response $h_{j}$ for the $j$ th transmitter can be determined, as $R_{x_{j} x_{j}}$ and $\rho$ are known. The earliest correlation peak that exceeds a particular threshold is corresponding to the direct propagation path from the DTV station to the position location receiver. The arrival time of the earliest correlation peak can then be converted to relative propagation time in terms of seconds. The correlation functions in (12) can be interpolated to improve the precision of the propagation time determined. The threshold for each DTV station is decided by the DTV station transmission power, the approximate distance between the DTV station and the receiver decided by the propagation time of the main path, and the maximum expected excess path loss to the DTV signal due to the building penetration.

The main path of the autocorrelation function in (12) is always used for transmitter identification due to its strongest signal power. The distance between the DTV station and the position location receiver depends only on the first arrived path. However, the strength of the first arrived signal sometimes is very weak, and it is difficult to discriminate multipath echoes from interference. In this case, the main path can always be used as a timing reference for averaging a number of adjacent transmitter identification results due to the slow variation of the DTV signals. Simple averaging of the transmitter identification results in the time domain would reduce the impact of the DTV interference by $10 \log _{10} P$, where $P$ is the number of averaging. The complexity associated with averaging is minimal since different DTV signal segments for TxID can be averaged first before the cross-correlation. An average of 42 fields of DTV signal within one second (168 Kasami sequence) will provide $22 \mathrm{~dB}$ gain. Very weak path such as $-30 \mathrm{~dB}$ echo can be easily identified when the 
averaging gain is imposed on SINR in (6). The impact of the interference on very weak first arrival echo is thus minimized. The number of averaging needed can be determined such that the noise power after averaging is below a predetermined threshold value, which is decided by the statistics of the interference in the transmitter identification results in (12). For Gaussian-like noise and interference, $10 \mathrm{~dB}$ below the threshold provide reliable decision. The averaging time is jointly determined by several factors, including DTV station transmission power level, the approximate distance (can be decided by the main lobe), and the maximum excess attenuation to the DTV signal due to penetration of building.

It is noted that (10) and (11) are ideal position location algorithm and no errors are taken into consideration. Under realistic conditions, a number of factors will introduce position location errors, including clock error for the DTV stations, synchronization errors between the DTV transmitter and position location receiver, nonideal shape of the autocorrelation peaks, multipath errors, and atmosphere errors. High accurate time and stable clock can be achieved from atomic clock, which minimize the impact of the clock error from the DTV stations. Atmosphere errors are out of control although some empirical models using dry and wet components can be used to remove some of them under given weather and geographic locations. In fact, atmosphere error is limited in the proposed position location system due to the short distance between the DTV stations and the receiver. Multipath errors due to weak strength of the first-arrived pre-echo can be minimized by time averaging of the transmitter identification results. The main echo of the multipath is always used as the reference to align different TxID correlation functions. As a result, nonideal shape of the correlation peak and time and frequency synchronization errors between DTV stations and the position location receivers are major sources of the position location process. The accuracy of the propagation time will be affected by the nonideal shape of the correlation peaks and timing offset of the receiver. Narrow and sharp correlation peak provides high time resolution and is less affected by interference. The strength of the correlation peak will be affected by frequency synchronization errors due to phase misalignment between the embedded TxID sequences and the local generated version.

\section{NONIDEAL CORRELATION FUNCTION}

In the previous analysis, the autocorrelation function of the transmitter identification watermark is approximated as a delta Kronecker function, which provides high time resolution for position location. However, the autocorrelation function shows a nonideal shape due to the bandlimitation of TV channels. It is important to analyze and compensate the bandlimitation effect in the transmitter identification results.

\subsection{Bandlimitation effect of DVB-T system}

Not all subcarriers are used in DVB-T systems to prevent adjacent channel interference. For example, in the DVB-T $2 \mathrm{k}$ mode, only 1706 of 2048 subcarriers are used. Under this circumstance, the baseband DVB-T signal can be reformulated as

$$
s(n)=\frac{1}{\sqrt{N}} \sum_{k=0}^{N-1} W_{k} S_{k} e^{j(2 \pi n k / N)}=w \otimes p,
$$

where

$$
\begin{aligned}
p & =\frac{1}{\sqrt{N}} \sum_{k=0}^{N-1} S_{k} e^{j(2 \pi n k / N)}, \\
W(k) & = \begin{cases}1, & k_{1} \leq k \leq k_{2}, \\
0, & \text { elsewhere, }\end{cases} \\
w & =\frac{1}{\sqrt{N}} \sum_{k=0}^{N-1} W_{k} e^{j(2 \pi n k / N)} \\
& =\frac{1}{\sqrt{N}} \frac{e^{j\left(2 \pi n\left(k_{1}+k_{2}\right) / N\right)} \sin \left(\pi n\left(k_{2}-k_{1}+1\right) / N\right)}{\sin (\pi n / N)} .
\end{aligned}
$$

Assume that the transmitter identification sequence has the same spectral mask as the DVB-T signal. The cross-correlation function between the embedded TxID sequence and the local reference now becomes

$$
R_{x^{\prime} x^{\prime}}(m)=\frac{1}{N} \sum_{n=0}^{N-1} x^{\prime}(n) x^{\prime *}(n-m)=R_{x x} \otimes R_{w w}
$$

where

$$
\begin{aligned}
R_{w w}(m) & =\frac{1}{N} \sum_{n=0}^{N-1} w(n) w^{*}(n-m) \\
& =\frac{1}{\sqrt{N}} \frac{e^{j\left(2 \pi m\left(k_{1}+k_{2}\right) / N\right)} \sin \left(\pi m\left(k_{2}-k_{1}+1\right) / N\right)}{\sin (\pi m / N)}
\end{aligned}
$$

Equation (17) indicates that each echo of impulse response identified by the TxID sequence is modulated by the shaping pulse in (18) due to the bandlimitation effect.

\subsection{Bandlimitation effect of ATSC system}

The ATSC 8-VSB modulator receives the $10.76 \mathrm{M}$ symbols/s, 8-level trellis encoded composite data signal (pilot and SYNC added) before it passes the VSB symbols to a root-raised cosine pulse shaping filter. The bandlimitation effect from the pulse shaping filer is to be analyzed in this section. The frequency response of the filter is essentially flat across the entire band, except for the transition regions at each end of the DTV signal. Nominally, the roll-off in the transmitter will have the response of a linear phase root-raised cosine filter 
according to

$$
W(\omega)= \begin{cases}1, & \omega<\omega_{c}(1-\alpha), \\ 0, & \omega>\omega_{c}(1-\alpha), \\ \sqrt{1+\cos \left(\frac{\pi\left[\omega-\omega_{c}(1-\alpha)\right]}{2 \alpha \omega_{c}}\right)}, \\ \omega_{c}(1-\alpha) \leq \omega \leq \omega_{c}(1+\alpha),\end{cases}
$$

where $\alpha$ is the roll-off factor of the raised cosine filter and $\omega_{c}$ is half the data rate in $\mathrm{rad} / \mathrm{sec}$. Since pulse filtering is equally split between the transmitter and the receiver, a pair of square-root cosine filters are often used. In theory, the response of the two cascaded square-root-raised cosine filters is equivalent to a single-raised cosine filter:

$$
W(\omega)=\left\{\begin{array}{lr}
1, & \omega<\omega_{c}(1-\alpha), \\
0, & \omega>\omega_{c}(1-\alpha), \\
1+\cos \left(\frac{\pi\left[\omega-\omega_{c}(1-\alpha)\right]}{2 \alpha \omega_{c}}\right), & \omega_{c}(1-\alpha) \leq \omega \leq \omega_{c}(1+\alpha) .
\end{array}\right.
$$

The impulse response of the filter in (15) is

$$
w(t)=\frac{\operatorname{sinc}(t / T) \cos (\pi \alpha t / T)}{1-4(\alpha t / T)^{2}} .
$$

However, the limited impulse response of practical squareroot-raised cosine filters causes a slight difference between the response of two successive square-root-raised cosine filters and the response of one raised cosine filter. The crosscorrelation function between the embedded TxID sequence and the local reference now becomes

$$
R_{x^{\prime} x^{\prime}}=R_{x x} \otimes R_{w w}
$$

\subsection{Compensation of the nonideal correlation function}

One possible way to resolve the problem is to eliminate the shape of the nonideal cross-correlation function from the preliminary channel estimation results. To simplify the notations, rewrite the channel estimation equation as

$$
R_{r x_{i}} \approx R_{w w}=\otimes h(n)+n^{\prime}(n)
$$

where $n^{\prime}(n)$ is the consolidated noise from the in-band DTV data signal and other interferences.

Let $\mathbf{w}=R_{w w}=[w(1), w(2), \ldots, w(L)]$. Rewrite the crosscorrelation between the received signal and pilot sequence $R_{r x_{i}}$ as vector $\mathbf{R}$ :

$$
\mathbf{R}=\mathbf{A h}+\mathbf{n}^{\prime},
$$

where

$$
\mathbf{A}=\left[\begin{array}{ccccc}
R_{w w}(L) & R_{w w}(L-1) & R_{w w}(L-2) & \cdots & R_{w w}(1) \\
R_{w w}(L+1) & R_{w w}(L) & R_{w w}(L-1) & \cdots & R_{w w}(2) \\
R_{w w}(L+2) & R_{w w}(L+1) & R_{w w}(L) & \cdots & R_{w w}(3) \\
\vdots & \vdots & \vdots & \ddots & \vdots \\
R_{w w}\left(L+L^{\prime}-1\right) & R_{w w}\left(L+L^{\prime}-2\right) & R_{w w}\left(L+L^{\prime}-3\right) & \cdots & R_{w w}(L)
\end{array}\right]
$$

when $\mathbf{n}^{\prime}$ is assumed to be Gaussian noise, $\mathbf{h}$ can be resolved using

$$
\mathbf{h}=\left(\mathbf{A}^{\mathrm{H}} \mathbf{A}\right)^{-1} \mathbf{A}^{\mathrm{H}} \mathbf{R},
$$

where $\mathbf{A}^{\mathbf{H}}$ is the hermitian of $\mathbf{A}$.

\section{TIME AND FREQUENCY SYNCHRONIZATION FOR THE POSITION LOCATION SYSTEM}

It is noted that transmitter identification sequence is synchronized with the DTV frame structure, since the time synchronization between the DTV signal $d(k)$ and the embedded transmitter identification code $x(k)$ can substantially re- duce the amount of the correlation computation during the identification process. Some time- and frequency-domain features of the DTV signal, for instance the ATSC PN511 sequence and the in-band pilots of DVB-T system, can be used for the timing and frequency synchronization purpose. Here the synchronization algorithm for ATSC is presented. Similar techniques can also be extended to DVB-T system using the time-domain sequence of the in-band pilots. The field sync in ATSC signal, that is, the PN-511 sequence in the $d(k)$, can provide an accurate starting point of TxID sequence using some autocorrelation techniques. In this case, cross-correlation in (8) is only to be computed during the delay spread of the transmitter impulse response. 
Denote the PN511 sequence as $p(n), n=0, \ldots, 511$, the timing synchronization process between the local TxID sequence in the received DTV signal and local TXID code is based on the cross-correlation between the received signal and the PN511

$$
R_{p r}(k)=\frac{1}{511} \sum_{n=0}^{510} p(n) r(n+k),
$$

where $k$ is the timing search range. For a satisfactory performance of the receiver, the first search range for the PN511 sequence has to be longer than the one for the DTV field. The largest correlation peak provides the synchronization time information. After the first acquisition of timing, the correlation range can then reduced to a range of several data symbols for the following correlation, in case there is only one transmitter.

Very often the receiver's clock is not locked to the frequency at the transmitter side, due to the substantial attenuation of the signal. The residual frequency offset due to the drifting of the local oscillator will definitely impact to the correlation function in (8). It is very common that an oscillator for the position location system may have a frequency offset up to several hundreds Herz. The destructive effect of the frequency offset is mainly because of the phase rotation of the data samples, which in fact reduces the effective TxID sequence length. The correlation peak will be reduced due the existence of the frequency offset.

Let $\Delta f$ be the frequency drifting for the local oscillator. Here we assume this offset remains unchanged during one ATSC field. We also assume an AWGN channel for the convenience of the analysis. The received signal becomes

$$
r(n)=[d(n)+\rho x(n)] \exp \left(j 2 \pi \Delta f n T_{s}\right)+n(n)
$$

The output from the channel estimation correlator is

$$
\begin{aligned}
R_{r x}(m) & =\frac{1}{N} \sum_{n=0}^{N-1} r(n) x^{*}(n-m) \\
& =\frac{1}{N} \sum_{n=0}^{N-1}\left(e^{j 2 \pi \Delta f n T_{s}} \cdot x(n) x(n-m)^{*}\right)+n^{\prime}(n) \\
& = \begin{cases}\frac{\sin \left(N \pi \Delta f T_{s}\right)}{N \sin \left(\pi \Delta f T_{s}\right)} R_{p p}+n^{\prime}(n), & m=0, \\
n^{\prime \prime}(n), & \text { elsewhere, }\end{cases}
\end{aligned}
$$

where

$$
\begin{aligned}
& n^{\prime}(n)=\frac{1}{N} \sum_{n=0}^{N-1}\left(e^{j 2 \pi \Delta f n T_{s}} \cdot d(n) x(n-m)^{*}\right)+n(n), \\
& n^{\prime \prime}(n)=\frac{1}{N} \sum_{n=0}^{N-1}\left(e^{j 2 \pi \Delta f n T_{s}} \cdot d(n) x(n-m)^{*}\right)+n(n) .
\end{aligned}
$$

It can be seen clearly from (29) that the main peak of the cross-correlation function in (8) now will be modulated by a sinc shaped function with its amplitude less than one. The maximum of the correlation function will be determined by the normalized frequency offset. That is the reason why the frequency offset has to be removed before the calculation of the propagation time between the transmitter and the receiver. The approach we proposed here for the estimation of the frequency drifting is based on the frequency-domain correlation between the received signal and the local TxID sequence after the timing synchronization is achieved. The implementation procedure for the proposed frequency offset estimation and compensation are as follows.

Step 1. Set the maximum of the frequency-domain correlation function $R_{\max }^{F}=0$.

Step 2. Create a complex TxID code signal as a local reference. This will generate the VSB modulated TxID signal $x_{\mathrm{VSB}}$ based on the local Kasami sequence.

Step 3. Compute $X^{*}(\omega)=\mathbb{F}\left(x_{\mathrm{VSB}}\right)^{*}$ where $\mathbb{F}$ is the Fourier transform operator and $*$ is the conjugate operator.

Step 4. For $\omega=\omega_{\text {nom }}-\omega_{\text {offset }}$ to $\omega=\omega_{\text {nom }}+\omega_{\text {offset }}$ with a step of $2 \omega_{\text {offset }} / L$ ( $L$ is the number of the searches).

(i) Compute the $R^{\prime}(\omega)$, which is the Fourier transform of one field of DTV signal modulated with a carrier frequency $\omega$, based on the timing synchronization information derived during the timing synchronization stage.

(ii) Obtain the frequency-domain correlation between the local TxID signal and the received signal,

$$
R_{p r}(\omega)=\frac{1}{N} \sum_{n=0}^{N} R^{\prime}(\omega) X^{*}(\omega) .
$$

Step 5. Upon exiting from the process, the frequency $\omega$ with maximum frequency-domain correlation is the estimated frequency offset.

Step 6. Remove the estimated frequency offset obtained in Step 5 from the received signal.

\section{NUMERICAL RESULTS}

Numerical simulations of the proposed transmitter identification system have been carried out. Code generator for Kasami sequence was developed in Matlab. Simulations of the transmitter identification and channel estimation using embedded Kasami sequence with period of $2^{16}-1$ have been carried out. Raised cosine pulse shaping and limited bandwidth effects were also included in this simulation. To guarantee that the DTV signal was not impaired by the TxID signal, the Kasami sequence was injected $30 \mathrm{~dB}$ below the DTV signal to prevent degradation as discussed earlier. A channel with a $6 \mathrm{~dB}$ and a $10 \mathrm{~dB}$ echoes was used for the desired transmitter. Simulation results are shown in Figure 4. 
It is observed that the dynamic range used for transmitter identification with $2^{16}-1$ Kasami sequences is only around $12 \mathrm{~dB}$ without any postprocessing. This dynamic range is good enough for transmitter identification, but may be low for channel estimation and low-level interference signal identification. Superimposition of the correlation functions can be used to improve the dynamic range, as this will smooth out the in-band DTV interference. A time-domain averaging technique was employed in Figure 4(b). The improvement in TxID dynamic range is calculated as $10 \log _{10} P \mathrm{~dB}$, where $P$ is the number of averaging times.

It is also noted that band pass filtering effects from the transmitter and receiver front ends are neglected in (4) for simplicity. In this case, the TxID results are in fact the convolution of the channel response in Figure 4(a) with the combined impulse response of transmitter and receiver front ends. For TxID purpose, Figure 4(b) is accurate enough, since only the strength of the main signal and strong multipath are to be identified. More precise channel estimation and interference identification may be obtained by reducing the bandlimit effects via deconvolution techniques, as indicated in Figure 4(c). The dynamic range in Figure 4(c) is about $30 \mathrm{~dB}$. It can be used to identify possible cochannel interference station that could have an impact to position location.

To verify the proposed position location system, three TV transmitters in Ottawa area were selected for the numerical simulations. The transmitter locations are shown in Figure 5. Here the timing reference is assumed to be known to the receiver. Therefore only three transmitters are needed to find out the three unknown parameters of the receiver's coordinates. These three transmitters are within forty kilometers from the Communications Research Centre. The GPS locations for these transmitters and the corresponding transmission power were assumed known to the receiver. The information was obtained through the Canadian television transmitter database from Industry Canada. Computer program was employed to simulate the signal propagation process. The GPS coordinate of the three transmitters are first converted to Cartesian coordinates $(x, y, z)$. The nonlinear equation system in (11) is solved using optimization techniques. Background noise was also injected. To simplify the analysis, free-space propagation models are used for all the three transmitters. The location results from the simulation were shown in Figure 6, where each star represents one round of location process. The accuracy of locationing process can be evaluated by the distance between the location results and the true location of the receiver (origin of the coordinates). The simulation results indicated that the accuracy of the proposed location system is within ten meters.

\section{CONCLUSIONS}

A new position location technique using the transmitter identification (TxID) sequences in the digital TV (DTV) signals was proposed. The principles of the transmitter identification system for ATSC and the proposed position locationing system were presented. Time and frequency synchronization between the receiver and DTV transmitter was

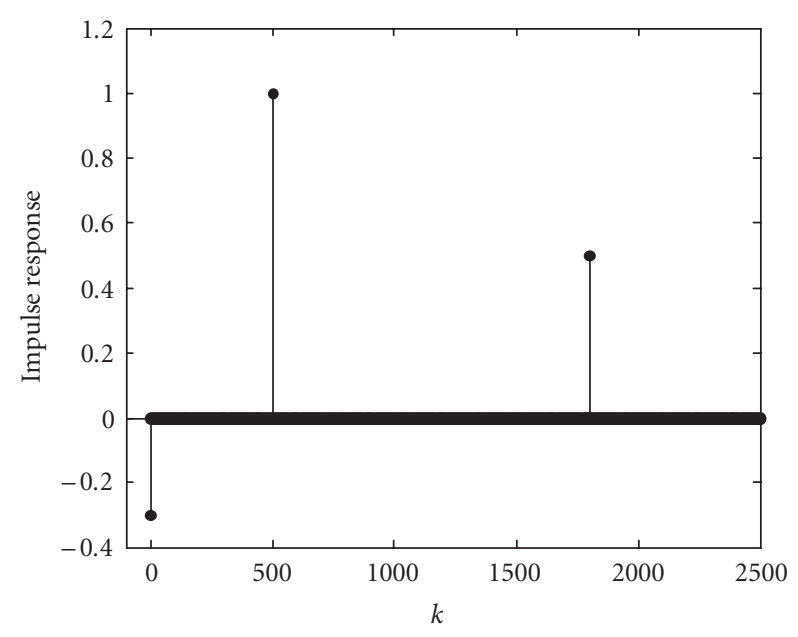

(a)

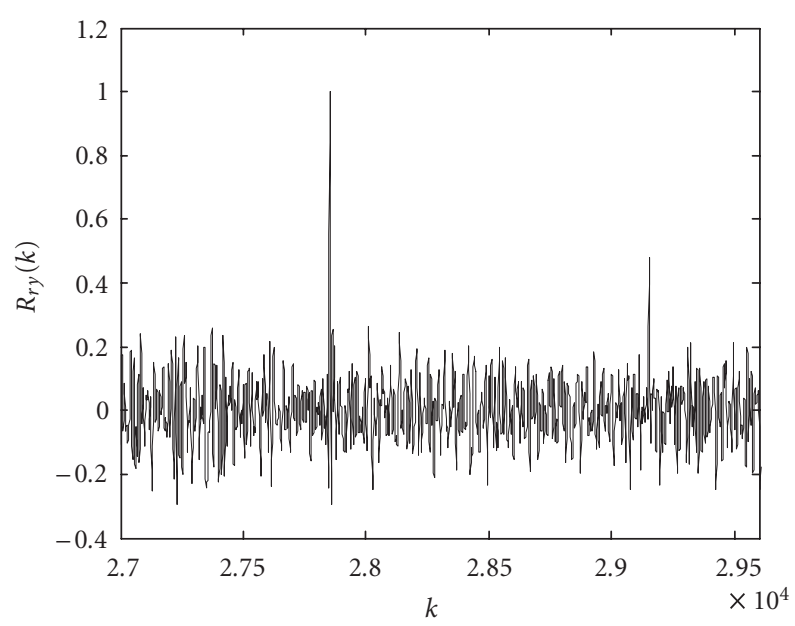

(b)

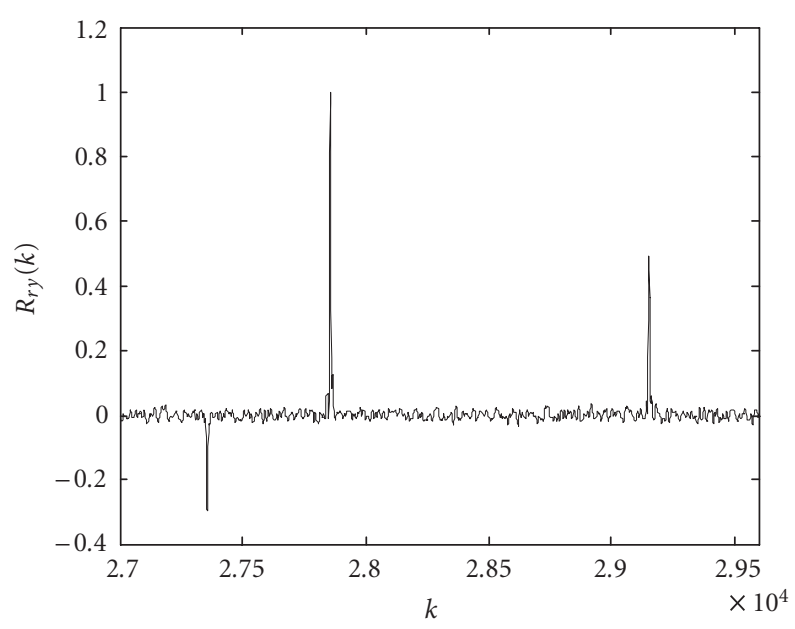

(c)

FIgURE 4: Example of ATSC transmitter identification using Kasami sequence. (a) Multipath used in the simulation, (b) identification results, (c) identification results after 60 times averaging. 


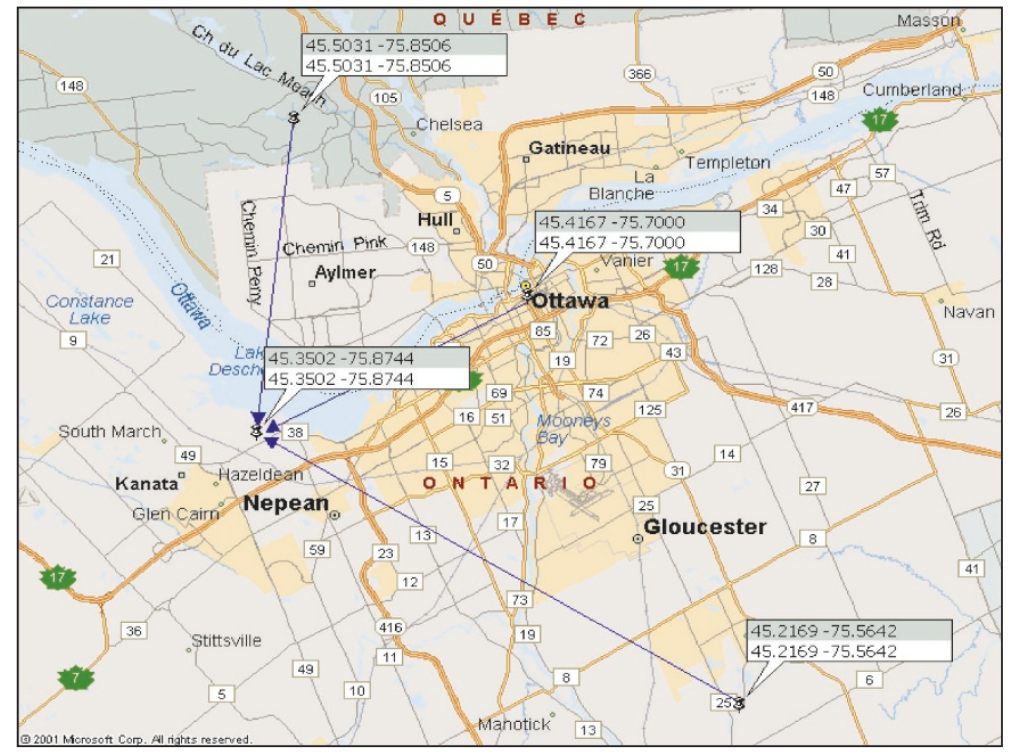

FIGURE 5: Locations of the transmitters used in the position location simulations.

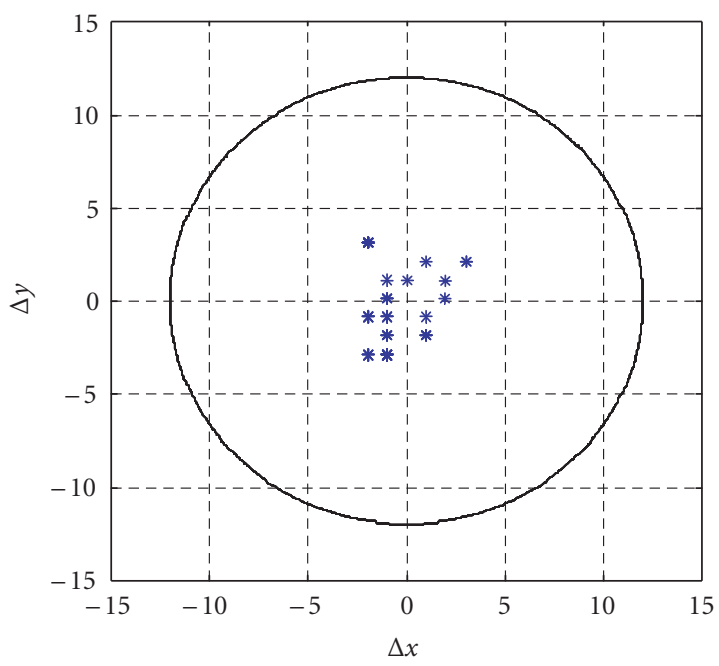

FIGURE 6: Numerical results for the proposed location position system based on TxID signal.

discussed. A new frequency-domain correlation technique was proposed to compensate the frequency drifting of the local oscillator. Performance of the proposed technique was evaluated through numerical simulations and compared with other existing position location systems. Possible ways to improve the accuracy of the new position location system were discussed.

\section{REFERENCES}

[1] E. D. Kaplan, Understanding GPS: Principles and Applications, Artech House, Norwood, Mass, USA, 1996.

[2] European Transport Policy for 2010: Time to Decide, European Commission, 2001.
[3] FCC Docket No. 94-102, “Revision of the Commission's Rules to Ensure Compatibility with Enhanced 911 Emergency Calling System," RM-8143, July 1996. (E-911).

[4] J. J. Caffery Jr. and G. L. Stuber, "Subscriber location in CDMA cellular networks," IEEE Transactions on Vehicular Technology, vol. 47, no. 2, pp. 406-416, 1998.

[5] J. J. Caffery Jr. and G. L. Stuber, "Overview of radiolocation in CDMA cellular systems," IEEE Communications Magazine, vol. 36, no. 4, pp. 38-45, 1998, Cellular Networks, Radiolocation Techniques.

[6] P. Prasithsangaree, P. Krishnamurthy, and P. K. Chrysanthis, "On indoor position location with wireless LANs," in The 13th IEEE International Symposium on Personal, Indoor and Mobile Radio Communications (PIMRC '02), vol. 2, pp. 720-724, Lisbon, Portugal, September 2002.

[7] M. Rabinowitz and J. J. Spilker Jr., "A new positioning system using television synchronization signals," IEEE Transactions on Broadcasting, vol. 51, no. 1, pp. 51-61, 2005.

[8] ATSC, ATSC Standard A/110: Synchronization Standard for Distributed Transmission, July 2004.

[9] X. Wang, Y. Wu, and B. Caron, "Transmitter identification using embedded pseudo random sequences," IEEE Transactions on Broadcasting, vol. 50, no. 3, pp. 244-252, 2004.

[10] R. E. Ziemer and R. L. Peterson, Digital Communications and Spread Spectrum Systems, Macmillan, New York, NY, USA, 1985.

[11] D. V. Sarwate and M. B. Pursley, "Cross correlation properties of pseudorandom and related sequences," Proceedings of the IEEE, vol. 68, no. 5, pp. 593-619, 1980.

[12] H. Hashemi, "The indoor radio propagation channel," Proceedings of the IEEE, vol. 81, no. 7, pp. 943-968, 1993.

[13] D. Molkdar, "Review on radio propagation into and within buildings," IEE Proceedings H: Microwaves, Antennas and Propagation, vol. 138, no. 1, pp. 61-73, 1991.

[14] A. Mattsson, "Single frequency networks in DTV," IEEE Transactions on Broadcasting, vol. 51, no. 4, pp. 413-422, 2005. 
[15] M. S. Grewal, L. R. Weill, and A. P. Andrews, Global Positioning Systems, Inertial Navigation, and Integration, John Wiley \& Sons, New York, NY, USA, 2001.

[16] J. A. Farrell and M. Barth, The Global Positioning System \& Inertial Navigation, McGraw-Hill, New York, NY, USA, 1999.

Xianbin Wang received his Ph.D. degree in electrical and computer engineering from the National University of Singapore, Singapore, in 2001. He was with the Institute for Infocomm Research, Singapore (formerly known as Centre for Wireless Communications), as a Senior R\&D Engineer in 2000. From December 2000 to July 2002, he was a system designer at STMicroelectronics Inc., where he was responsible for system de-

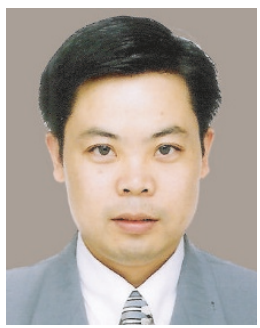
sign for DSL and gigabit ethernet chipsets. Since July 2002, he has been with the Communications Research Centre Canada, Ottawa, Canada, where he is currently a Senior Research Scientist. $\mathrm{He}$ is also an Adjunct Associate Professor at Laval University, QC, Canada. His current research interests include digital signal processing, broadband wireless system, and communication theory. $\mathrm{He}$ is the recipient of the IEEE Scott Helt Memorial Award for the Best Paper published in IEEE Transactions on Broadcasting in 2004.

Yiyan Wu received the M.Eng. and Ph.D. degrees in electrical engineering from Carleton University, Ottawa, Canada, in 1986 and 1990, respectively. After graduation, he worked at Telesat Canada as a Senior satellite communication systems Engineer. In 1992, he joined Communications Research Centre Canada (CRC) and is now a Principle Research Scientist. His research interests include broadband multimedia communi-

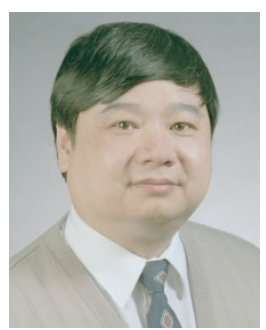
cations, digital broadcasting, and communication systems engineering. He is an IEEE Fellow, an Adjunct Professor of Carleton University, Ottawa, Canada. He is a Member of the IEEE Broadcast Technology Society Administrative Committee, and a Member of the ATSC Board of Directors, representing the IEEE. He is the Editor-in-Chief of the IEEE Transactions on Broadcasting. He has more than 200 publications and received many technical awards for his contribution to the research and development of digital broadcasting and broadband multimedia communications.

Jean-Yves Chouinard received the B.S.A., M.S., and Ph.D. degrees in electrical engineering from Laval University in 1979, 1984, and 1987, respectively. From 1979 to 1981, he was with Northern Telecom in Montreal. From 1987 to 1988, he was a postdoctoral fellow at the Space and Radio Communication Division of the Centre National d'Études des Télécommunications (CNET) in France. From 1988 to 2002, he

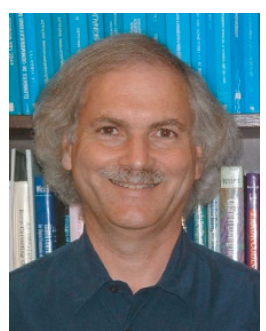
was with the School of Information Technology and Engineering (SITE) at the University of Ottawa. From 1996 to 1997, he was an Invited Professor at the École Nationale Supérieure des Télécommunications (ENST) in France. Since 2003, he is with the Department of Electrical and Computer Engineering at Laval University in Quebec. His research interests are communication theory and applications, wideband mobile and indoor wireless systems, digital channel modelling, error control coding and digital modulation techniques for advanced television systems. He is an IEEE Senior Member and a Member of the Ordre des Ingénieurs du Québec (OIQ) and of the Canadian Society for Electrical and Computer Engineering (CSECE). He is an Associate Editor for the IEEE Transactions on Broadcasting. He is also a Director of the Canadian Society of Information Theory (CSIT). 